\title{
Liebe und Schmerz als Schlüssel zur Gefühlswelt
}

\author{
K. E. Schneider
}

\footnotetext{
* In diesem Text werden die Begriffe Gefühl, Emotion und Affekt als Synonyme verwendet.
}

1 Käsermann M-L, et al. Nationalfondsprojekte 1113 und 1114.

2 Schneider KE. Liebe \& Schmerz. Ein Schlüssel zur Gefühlswelt. Basel: Schwabe \& Co. AG; 2002.

3 Strongman K. The psychology of emotion. Chichester: Wiley \& Son; 1987.

4 Lempp R. Vom Verlust der Fähigkeit, sich selbst zu betrachten. Eine entwicklungspsychologische Erklärung der Schizophrenie und des Autismus. Bern: Hans Huber; 1992.

5 Tart CT. Open Mind, Discriminating Mind. San Francisco: Harper \& Row; 1989.

Korrespondenz:

Dr. med. Kurt. E. Schneider

St. Alban-Vorstadt 80

4052 Basel
In letzter Zeit hat die wissenschaftliche Forschung - nicht erst im Zusammenhang mit dem grossen Interesse, das die emotionale Intelligenz öffentlich erregte - zunehmend die verschiedenen Formen des gefühlsmässigen Ausdrucks sowie der emotionalen Biologie unter die Lupe genommen. Seit dem Jahr 2000 gibt die American Psychological Association APA die Vierteljahreszeitschrift Emotion heraus, und sogar der Schweizerische Nationalfonds unterstützt ein Projekt über emotionale Prozesse, welches von der psychiatrischen Klinik der Uni Bern [1] durchgeführt wird. Die Gefühle* nehmen insbesondere als Modulatoren und Vermittler zwischen Körper und Verstand einen zentralen Platz ein [2].

Abgesehen von der generell akzeptierten Einteilung in positive und negative Gefühle gibt es noch keine Theorie, welche die verschiedenen Emotionen in einem Modell klassifiziert (vgl. dazu Strongman [3], der über dreiunddreissig emotionale Theorien auflistet).

Der folgende Ansatz [2] versucht diesem Ziel näher zu kommen; er stellt vier Gefühlsebenen (Körperebene, zwei psychische Ebenen des Primärselbst respektive des Sekundärselbst sowie der spirituellen Ebene; Tab. 1), fünf emotionale Bausteine (Tab. 2) gegenüber.

Um diese neue Perspektive zu ermöglichen, werden Bereiche in die Diskussion miteinbezogen, welche bisher kein wissenschaftliches Interesse gefunden haben. Dies bedeutet, um es im Kontext der Realitätsfrage auszudrücken, dass das Augenmerk nicht mehr ausschliesslich auf die wissenschaftlich sanktionierte Hauptrealität gerichtet, sondern dass auch bestimmte «Nebenrealitäten» [4] einer Betrachtung für würdig befunden werden.

Die Realitätsfrage ist eng mit der Sozialisation verknüpft: Während erwachsene Menschen innerhalb der gegebenen Gesellschaften - welche sich «globalisiert» immer mehr mit den Werten der westlichen Weh identifizieren - in Bezug auf das, was ihre Realität ausmacht, weitgehend einig sind, leben nicht nur sogenannt primitive Kulturen, sondern viele Individuen und besonders Kinder manchmal oder sogar meistens in einer eigenen «Realität». Innerhalb einer gegebenen Zivilisation existiert eine Hauptrealität
(Tart spricht von Konsensus Trance [5]); daneben gibt es aber unendlich viele Nebenrealitäten. Viele dieser Nebenrealitäten können als bedrohlich angesehen werden, wie beispielsweise im religiösen Bereich oder im Zusammenhang mit veränderten Bewusstseinszuständen (ASC). Dies gilt erst recht für psychopathologische Nebenrealitäten (Wahn und Psychosen).

Wird die Realitätsfrage miteinbezogen, so zeigt es sich, dass neu auch die Gefühle verschiedenen «Wirklichkeiten» zugeordnet werden müssen:

Die Körperebene und die psychische Ebene des sozialisierten Sekundärselbst entsprechen unserer Hauptrealität, während die psychische Ebene des präsozialisierten Primärselbst sowie die spirituelle Ebene (Zenrealität oder kosmische Realität) wissenschaftlich gesehen als «irreal» eingestuft werden und deshalb von der emotionalen Forschung nicht ernstgenommen werden. Trotzdem zeigt es sich, dass die Zenrealität einer universalen Nebenrealität entspricht, indem diese auch die Sekundärselbstebene umfasst, weil es nicht möglich ist, zur bewussten Wirklichkeit des Zen vorzudringen, ohne die Zwischenstufe der Sozialisation gelebt zu haben. Aus diesem Grund ist es für «Zenreale» möglich, im Umkehrprozess - Lempp spricht von Überstieg [4] - zurück zur hauptrealen Sekundärselbstebene zu transgredieren.

Die vier Gefühlsebenen unterscheiden sich vorwiegend auf Grund der folgenden Kriterien: «Selbst», «Realität», «Bewusstsein», und «Gefühle» (siehe Tabelle 1).

Indem wir diese vier Ebenen mit den fünf Gefühlsbausteinen (Seinsliebe, Energie, Schmerz, Kampf! Flucht Reflex und Sozialisation) in Bezug setzen, ergibt sich ein Raster innerhalb dessen sich die grundlegenden Emotionen zuordnen lassen (siehe Tabelle 2).

Im folgenden werden die in den Tabellen 1 und 2 verwendeten Begriffe näher erläutert.

Die Seinsliebe wird als homöostatischer Basiszustand aller höheren Lebewesen, inklusive des Menschen, definiert. Wesentliche Qualitäten dieses emotionalen Ruhezustandes sind Offenheit, Kritiklosigkeit, Zeit- und Raumlosigkeit, eutoner Muskelzustand, aperspektivische Erfahrungswelt u.a. 
Tabelle 1

Die vier Gefühlsebenen.

\begin{tabular}{|c|c|c|c|c|}
\hline \multirow[t]{2}{*}{ Ebene } & \multicolumn{3}{|l|}{ Körperebene } & \multirow{2}{*}{$\begin{array}{l}\text { spirituelle Ebene } \\
4\end{array}$} \\
\hline & 1 & 2 & 3 & \\
\hline «Selbst» & Wesen & Primärselbst & Sekundärselbst (Ich/Ego) & «Nicht-Selbst» \\
\hline \multirow[t]{3}{*}{ «Realität» } & Hauptrealität & Nebenrealität & Hauptrealität & Nebenrealität, Zenrealität, «kosmische R.» \\
\hline & & - pränatal & & \\
\hline & & - Säugling & & \\
\hline «Bewusstsein» & vorbewusst & unbewusst (offen) & «bewusst» (programmiert) & bewusst (offen) \\
\hline «Gefühle» & Körpererfahrung & (psychische) Primärgefühle & (psychische) Sekundärgefühle & Energiewahrnehmung \\
\hline
\end{tabular}

Tabelle 2

Die fünf Gefühlsbausteine in Abhängigkeit von den Gefühlsebenen.

\begin{tabular}{|c|c|c|c|c|}
\hline \multirow[t]{2}{*}{ Gefühlsebene } & \multirow[t]{2}{*}{ Körperebene } & \multicolumn{2}{|l|}{ Psychische Ebenen } & \multirow[t]{2}{*}{ Spirituelle Ebene } \\
\hline & & Primärselbst & Sekundärselbst & \\
\hline & 1 & 2 & 3 & 4 \\
\hline Sozialisation & Instinkt & präsozialisiert & sozialisiert & de-sozialisiert \\
\hline Seinsliebe & Sein & unbewusste Seinsliebe & Zweckliebe & bewusste Seinsliebe \\
\hline Energie & $\begin{array}{l}\text { Erregung (sexuell) Tanz, } \\
\text { Lachen usw. }\end{array}$ & primäre Erregungsliebe & $\begin{array}{l}\text { sekundäre Erregungsliebe } \\
\text { Phantasie, Ersatz }\end{array}$ & Energiewahrnehmung Tantra, Ekstase \\
\hline \multirow[t]{2}{*}{ Schmerz } & Gewebeschaden & Liebesentzug & Kränkung & Körperschmerz \\
\hline & - Körperschmerz & - psychischer Primärschmerz & - psychischer Sekundärschmerz & (psych. Schmerzen «durchschaut») \\
\hline \multirow[t]{3}{*}{ Kampf/Flucht } & Verletzung/Bedrohung & & & \\
\hline & - Kampf & psychische Primärwut & $\begin{array}{l}\text { psychische Sekundärwut } \\
\text { (Hass, Gewalt) }\end{array}$ & «Überstieg» als Option \\
\hline & - Flucht & psychische Primärangst & $\begin{array}{l}\text { psychische Sekundärängste } \\
\text { (Phobien, Zwänge etc.) }\end{array}$ & \\
\hline
\end{tabular}

Diese These mag viele Leser befremden, sie ist aber von fundamentaler Bedeutung und wird im Buch «Liebe \& Schmerz» [2] ausführlich diskutiert. Wegen der zentralen Bedeutung der Liebe für das Verständnis der menschlichen Emotionen ist es zwingend, dieses vieldeutige Wort möglichst genau zu beschreiben; es werden vier verschiedene Aspekte unterschieden: Der nichterregte Basiszustand der Seinsliebe und die primäre Erregungsliebe, beide im Rahmen des präsozialisierten Primärselbst, sowie - als hauptreale Sekundärselbstaspekte die basale Zweckliebe und die sekundäre Erregungsliebe (Phantasie).

Im emotionalen Labor wird der Erregung, welche indirekt über die Messung von Puls, Blutdruck oder Hautwiderstand registriert wird, ein immer grösseres Gewicht beigemessen. Dass aber Energie (in seiner alläglichen Bedeutung, d.h. nicht nur rein physikalisch gesehen) als entscheidender Faktor eingeführt wird, mag viele erstaunen.
Der Hintergrund $\mathrm{zu}$ diesem Schritt ergibt sich aus der Meditationserfahrung, wie sie in östlichen Kulturen seit über dreitausend Jahren praktiziert wird. Dabei wird, über die zunehmende Desidentifikation von den Sozialisationsprogrammen, der Affekt schliesslich als reines «Energie»-Phänomen erlebt; unsere Verhaltensmuster aus der frühesten Kindheit sowie die vielen fremdbestimmten Erziehungsprogramme werden durchschaut und im unmittelbaren Gegenwartsbezug hinterfragt.

Steigt nun aus dem Basiszustand der Seinsliebe - aus was für äusseren oder inneren Ursachen heraus - die «Energie» an, so sind die Voraussetzungen für die primäre Erregungsliebe gegeben. Diese ist das positive Grundgefühl, das sich entweder unmittelbar ausdrückt oder, als potentielle Energie gespeichert, den ganzen Organismus belebt (beispielsweise im Kontext des Talorgasmus). 
Abbildung 1

Die Verdrängungskaskade.

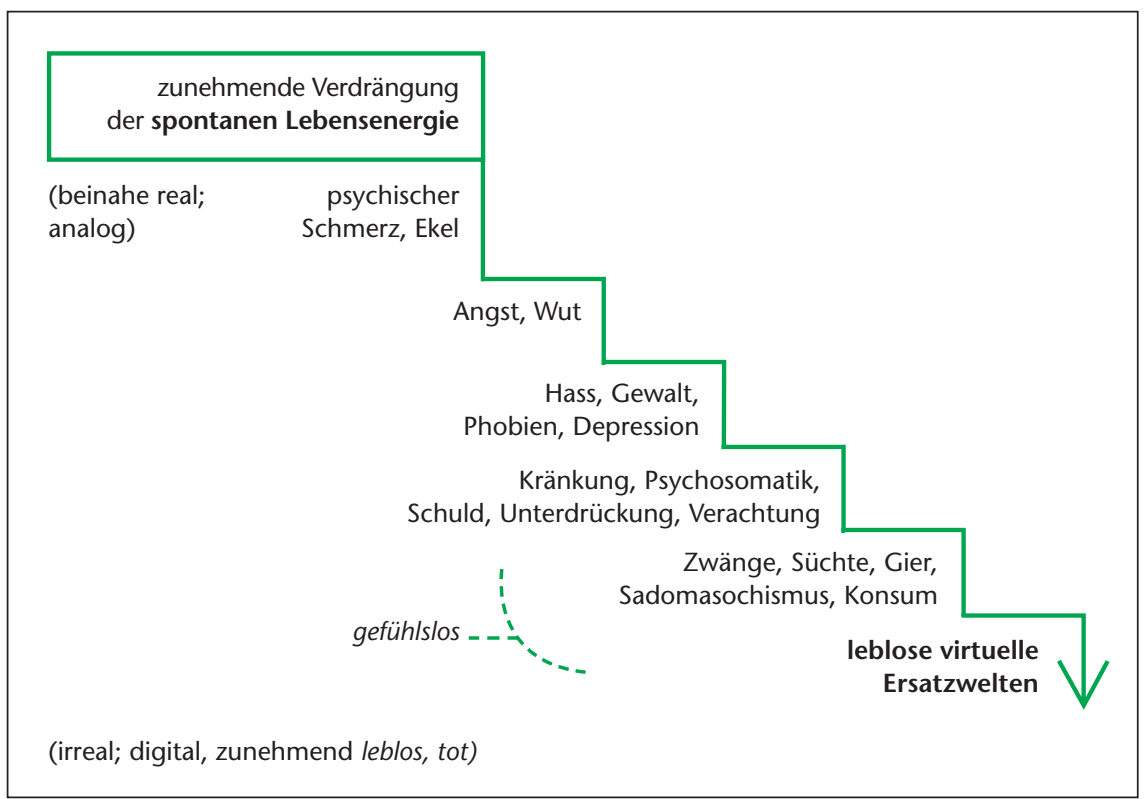

Wird dieser als lustvoll erfahrene Ausdruck gehemmt oder unterdrückt, so ist die unmittelbare Antwort darauf psychisch empfundener Schmerz.

Der Schmerz wird selten in die Gefühlsforschung einbezogen. Die meisten Wissenschafter erwähnen ihn nicht, ähnlich wie auch die «Liebe» häufig übergangen wird. Für mich ist Schmerz die unmittelbare Reaktion nicht nur auf zelluläre Schäden, sondern auch - in seiner psychischen Form - auf Liebesentzug respektive auf die durch äussere oder innere Umstände (Hemmung/Verbot) erwirkte Unterdrückung.

Je nach der Gefühlsebene lassen sich beim Schmerz vier verschiedene Varianten beschreiben: Der physisch-organische Schmerz, welchen wohl die meisten Lebewesen in irgendeiner Form empfinden, psychische, noch stark mit Körperreaktionen (Verspannungen u.a.) verknüpfte Primärschmerz, welcher den (drohenden) Verlust zu einer existentiell bedeutungsvollen «lieben» Person mit sich bringt, psychischen Sekundärschmerzen, welche sich aus (Ego-)Kränkungen und sozialisationsbedingten Hemmechanismen heraus ergeben, sowie die Abwesenheit psychischer Schmerzen im Zustand der Zenrealität. Weil insbesondere die psychischen Sekundärschmerzen erst auf Grund von Erinnerungen oder von vorgefassten Meinungen entstehen, und damit «selbstgemacht» sind, werden nur noch körperliche Schmerzen als «schmerzhaft» wahrgenommen. Im Idealfall verschwinden auch die psychischen Primärschmerzen, weil sich frühe traumatische Muskelreaktionen als Folge der tiefen Meditationsstufe auflösen.

Der Schmerz und seine unmittelbaren Folgen sind Teil des sensomotorischen Systems, welches sich - anatomisch gesehen - aus der epidermalen und mesodermalen Embryonalschicht entwickelt. Brechreiz und Ekel sind körperliche und psychische Reaktionen auf Läsionen oder unmittelbar bevorstehende chemisch/toxische Schädigung des intestinalen entodermalen Systems; sie sind gefühlsmässig mit der Körperebene und der Primärselbstebene verknüpft.

Scham und psychisch verursachte Ekelgefühle sowie Schuld gehören der Ebene des Sekundärselbst an, ähnlich wie Verachtung, Stolz/Arroganz oder Selbstverachtung, welche, bezogen auf die Verdrängungskaskade, noch weiterentwickelten (sozialisierten) Gefühlen entsprechen. Diese zunehmend komplexeren intestinalen Emotionen vermischen sich immer mehr mit den Sekundärgefühlen der sensomotorischen Reihe.

Sowohl Wut als auch Angst sind instinktmässige Verhaltensweisen auf die Erfahrung von physischem oder psychischem Schmerz respektive auf entsprechende Bedrohung; sie stehen in unmittelbarem Zusammenhang mit dem Kampf/ Flucht-Reflex.

Beide Gefühle manifestieren sich, in ihrer körperlichen sowie der Primärselbstform, spontan und deutlich als Aggression oder Rückzug, primäre Wut oder primäre Angst. Im Zusammenhang mit den komplizierten und berechnenden Operationen des (Sekundärselbst-)Verstandes kann sich Wut als mörderischer Hass oder tödliche Gewalt ausdrücken, Angst in die verschiedenartigsten sozialen Phobien, neurotischen Zwänge oder gar in Depression ausarten.

Den Auswirkungen der Sozialisation auf die menschlichen Gefühle kommt eine sehr grosse, wenn auch oft verkannte oder gar bewusst ignorierte Bedeutung zu.

Dieser Einfluss lässt sich besonders deutlich im Zusammenhang mit den Diskussionen zum Selbst nachweisen: Auch wenn dies nicht allgemein anerkannt ist, so besteht für mich kein Zweifel, dass es notwendig ist, ein Primärselbst von einem sozialisierten erwachsenen (hauptrealen) Sekundärselbst abzugrenzen, einem Selbst, das sich enorm vom pränatalen (perspektivelosen) und frühkindlichen (analogen) Primärselbst unterscheidet.

Daraus abgeleitet gilt es jetzt, immer noch im Zusammenhang mit den Auswirkungen der Sozialisierung, auch zwei ganz verschiedene 
Emotionsformen zu unterscheiden: Primärgefühle, spontan, unmittelbar körperbezogen und ursprünglich sowie Sekundärgefühle, welche durch kognitive (digitale) Verstandesprozesse, Phantasien, Wunschdenken und Vorstellungen angereichert sind.

Die Verdrängungskaskade (Abb. 1) ist ein allgegenwärtiger psychischer Mechanismus des «Nicht-Wahrhaben-Wollens», um mit den nagenden Gefühlen der psychischen Schmerzen «fertig» zu werden.

Über zunehmend komplexere und mental veränderte Sekundärgefühle bis zum Gefühlsersatz verdrängt der moderne Mensch seine Affekte hin zu immer abstrakteren, körperfernen und schliesslich unterkühlt leblosen Bereichen. Bevor das Endstadium der Athymie erreicht ist, treffen wir auf verschiedene Übergangsformen: sadomasochistische Praktiken, unterschiedlichste Zwangs- und Suchtformen, unersättliche Habgier (vom Geld über Prestige und Macht bis zum Überkonsum) sowie workaholische Arbeitswut. Der Prozess der Verdrängung erweist sich als ein Fass ohne Boden mit zunehmender Beschleunigung und vielen Vermarktungsmöglichkeiten.

Zusammenfassend wird ein neues Konzept vorgestellt, welches die Entstehung und die Modifikation der menschlichen Gefühle modellhaft aufzeigt.

Neu werden Erkenntnisse berücksichtigt, welche sich aus einer Öffnung gegenüber der Nebenrealität des unbewussten Primärselbst auf der einen Seite sowie gegenüber der nachsozialisierten Zenrealität (im Zustand des bewussten Primärselbst) auf der anderen Seite des hauptrealen (erwachsenen) Sekundärselbst ergeben. Dadurch gewinnen wir Zugang zu einer weitergefassten Übersicht der menschlichen Gefühle.

Zentrale Fixpunkte in dieser Theorie sind die Definition eines liebevollen Basiszustandes, genannt Seinsliebe, sowie der Einbezug der energetischen Erregung als prinzipiellen Motors der Emotionen. Die als lustvoll empfundene primäre Erregungsliebe verkörpert das positive Grundgefühl. Unter dem Einfluss der Sozialisation entsteht daraus der Basiszustand der Zweckliebe sowie die über Wunschvorstellungen modifizierte sekundäre Erregungsliebe.

Der Schmerz ist, zumindest innerhalb der sensomotorischen Gefühle, der zentrale negative Affekt, aus dem heraus sich - modifiziert durch den primitiven Kampf/Flucht-Reflex sowie durch die höchst differenzierten und komplexen Sozialisationseinflüsse - alle anderen (negativen) Emotionen ableiten. Innerhalb der zuletzt genannten Einflüsse spielt die Bewältigungsstrategie der Verdrängung eine dominierende Rolle. Über die Verdrängungskaskade versuchen wir, psychische Schmerzen zu umgehen; ihr vorläufiges Ende lässt sich als eine virtuelle Kunstwelt, die sich von unmittelbar körperlichen Bezügen gelöst hat, nicht nur auf dem Bildschirm, sondern bereits in den zwischenmenschlichen Beziehungen nachweisen. 